treatment interruptions and/or reduced dose were at risk of relapse.

Disclosure(s) Nothing to disclose

\section{P04 NON-COMPARTMENTAL ANALYSIS OF VANCOMYCIN PHARMACOKINETICS IN CRITICALLY ILL CHILDREN}

${ }^{1} \mathrm{O}$ Akunne ${ }^{*},{ }^{1} \mathrm{P}$ Mugabo, ${ }^{2,3} \mathrm{~A}$ Argent. ${ }^{1}$ Discipline of Pharmacology, School of Pharmacy, University of the Western Cape, Bellville; ${ }^{2}$ Paediatrics and Child Health, University of Cape Town; ${ }^{3}$ Paediatric Intensive and Critical Unit, Red Cross War Memorial Children Hospital, Cape Town, South Africa

\subsection{6/archdischild-2019-esdppp.43}

Background Vancomycin is often used as the drug of choice in the treatment of bacteria that are methicillin resistant or in patients sensitive to penicillin. This study describes the pharmacokinetics of vancomycin in critically ill children.

Methods Children aged 1month to 16 years admitted to the paediatric intensive care unit of the Red Cross War Memorial Hospital and on vancomycin treatment for $\geq 24$ hours and $\leq 72$ hours were prospectively recruited. Blood samples were collected around predetermined optimal sampling times. A minimum of three samples per patients was analysed. Non-compartmental analysis was used to determine the volume of distribution (V), clearance $(\mathrm{CL})$, half-life $\left(\mathrm{t}_{1 / 2}\right)$, area under the concentration-time curve (AUC), elimination constant (Ke) and Mean residence time (MRT). The minimum concentration (Cmin) and maximum concentration (Cmax) of vancomycin were measured directly as trough and peak plasma concentration respectively. Analysis of data was performed using PKNCA version 0.8.5 in R.

Results Forty-nine serum concentrations from 10 patients were included in the analysis. The ratio of male to female was $1: 1$. Median age (Range) was $1.6(0.2-15)$ years, weight $=10.6$ $(3.1-31.3) \mathrm{kg}$, baseline serum creatinine $(\mathrm{Scr})=0.31(0.15-$ $0.78) \mathrm{mg} / \mathrm{dL}$. Patients received daily vancomycin doses ranging from 56 to $78 \mathrm{mg} / \mathrm{kg}$. Mean PK parameters (range) were as follows: $\mathrm{CL}=0.12 \pm 0.15(0.018-0.52) \mathrm{L} / \mathrm{h} / \mathrm{kg}, \mathrm{V}=0.68$ $\pm 0.47(0.15-1.57) \mathrm{L} / \mathrm{kg}, \mathrm{Ke}=0.147 \pm 0.073(0.07-0.33) \mathrm{h}^{-1}$, $\mathrm{t}_{1 / 2}=5.54 \pm 2.03 \quad(2.11-9.62) \quad \mathrm{h}, \quad \mathrm{MRT}=8.02 \pm 2.92 \quad(3.04-$ $13.88) \mathrm{h}$, AUC $=322.07 \pm 245.65(31.6-850.4) \mathrm{mg} / \mathrm{L} . \mathrm{h}, \mathrm{Cmin}$ $=6.21 \pm 3.66(2.0-14.5) \mathrm{mg}, \mathrm{Cmax}=38.91 \pm 31.92(10.4-97.2)$ mg. Trough concentration and AUC were not met in $>80 \%$ and $>75 \%$ of the patients.

Conclusion Variability in vancomycin pharmacokinetics was observed in patients. At current doses, target trough concentrations and AUC were not met.

Disclosure(s) Nothing to disclose

\section{P05 ESTABLISHING THE INTEGRITY OF THE CONTINUALLY PAEDIATRIC PHARMACOKINETIC BIOANALYSIS FOR CLINICAL TRIAL WITHIN AN ACADEMIA ENVIRONMENT USING QUALITY ASSESSMENT PROCESS}

M Ali*, J Tins, BB Burckhardt. Institute for Clinical Pharmacy and Pharmacotherapy, Heinrich Heine University, Duesseldorf, Germany

10.1136/archdischild-2019-esdppp.44

Background European Medicines Agency (EMA) outlines criterion for validation of bioanalytical assay applied under Good Clinical Laboratory Practice (GCLP) within clinical studies. The validation is performed once and does not inevitably ensures comparable reliability over long duration of assay's applicability. To address this hurdle, the investigator-driven 'Labelling of Enalapril from Neonates up to Adolescents' project (LENA) adapted quality system comparable to industry. A comprehensive set of quality measures was applied to ensure reliability of monthly quantified paediatric samples over duration of 31 months.

Methods A 3-step quality approach analysing the calibration standards (CS), quality controls (QCs) and incurred sample reanalysis (ISR) was used to established reliability of unknown samples. Unknown concentrations were reported only if results of known CS (11 levels) and QCs were within the predefined limits. A maximum deviation of $\pm 15 \%$ was acceptable at all five QCs level. ISR was conducted for randomly selected paediatric samples to monitor the assay's performance over time. The acceptable $\%$ difference was $\pm 20 \%$ for at least $67 \%$ of the ISR according to international guidelines. ${ }^{1,2}$

Results 38 analytical runs were conducted for two drugs (enalapril/enalaprilat) from February 2016 to August 2018. Calibration curve evaluation accounted for exclusion of four enalapril and five enalaprilat runs. Additional investigation of QCs resulted in further exclusion of two enalapril and one enalaprilat runs. Within 32 valid runs 820, QCs were measured for enalapril and enalaprilat. $94 \%$ enalapril and $89 \%$ enalaprilat QCs were within limits $( \pm 15 \%)$. This set ensured reliable determination of 1262 LENA paediatric study samples. 93 and 104 incurred samples for enalapril and enalaprilat were reanalysed. ISR for enalapril (70\%) and enalaprilat $(67 \%)$ was also within guidelines. ${ }^{1,2}$

Conclusion The analysis of bioanalytical data ensured the reliability of reported unknown concentrations. It ensured consistent and reliable pharmacokinetic data from first to the last LENA study patient.

\section{REFERENCES}

1. Guideline on bioanalytical method validation. European Medicines Agency, London, UK (2011).

2. Guidance for Industry Bioanalytical Method Validation. US Department of Health and Human Services, US FDA, Rockville, MD, USA (2018).

Disclosure(s) Mohsin Ali, Jutta Tins, Bjoern B Burckhardt declare that there is no conflict of interest. The research leading to these results has received funding from the European Union Seventh Framework Programme (FP7/2007-2013) under grant agreement $n^{\circ} 602295$ (LENA)

\section{P06 PATERNAL EXPOSURE TO METHOTREXATE AND THE RISK OF MISCARRIAGE - A REGISTER BASED NATIONWIDE COHORT STUDY}

\footnotetext{
1,2J Andersen*, ${ }^{3} \mathrm{~B}$ Askaa, ${ }^{1} \mathrm{~TB}$ Jensen, ${ }^{1,2} \mathrm{H}$ Horwitz, ${ }^{1,2} \mathrm{C}$ Vermehren, ${ }^{1} \mathrm{~K}$ Broedbaek. ${ }^{1}$ Copenhagen University Hospital Bispebjerg; ${ }^{2}$ University of Copenhagen; ${ }^{3}$ Copenhagen University Hospital Rigshospitalet, Copenhagen, Denmark
}

10.1136/archdischild-2019-esdppp.45

Objective To study the association between paternal exposure to methotrexate within three month before conception and during the first trimester of pregnancy and the risk of miscarriage.

Methods We conducted a nationwide cohort study identifying all registered pregnancies in Denmark from 1997 to 2015. All births were identified using the Medical Birth Registry, and all records of induced abortion and miscarriage were from the National Hospital Register. Data on drug use were from the 